ctive.

推论 1 若 Locale $\boldsymbol{A}$ 有 Flat sublocale $A_{i}$ 是 Connective, 则 $A$ 也是 Connective.

定理 4 Spatial Locale $A$ 是 Connective 当且仅当拓扑空间 Spec $(A)$ 是连通 空间.

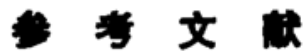

[1] Johnstone, P. T., Stone Spaces, Cambridge Univ. Press, Cambridge, 1982.

白仲林

(西北师范大学数学系, 兰州 730070)

\title{
关于 $x+y_{2} \rightarrow x y+y$ 反应的速率常数的计算
}

体系的过渡态是直线型的, 有效势能为

$$
U\left(r_{1}\right)=V\left(r_{1}\right)+\frac{l(l+1) \hbar^{2}}{2 I\left(r_{1}\right)},
$$

$l$ 为转动量子数, $M_{1}$ 和 $M_{2}$ 分别是 $\mathrm{y}$ 原子和 $\mathrm{x}$ 原子的质量, $r_{1}$ 和 $r_{2}$ 分别是 $\mathrm{y}$ 原子间及 $x$ 与较近的一个 $y$ 原子间的距离. 体系对于质 心的转动惯量是

$$
\begin{aligned}
I\left(r_{1}\right)= & \left\{2 M_{1} M_{2} r_{2}^{2}+\left(M_{1}+M_{2}\right) M_{1} r_{1}^{2}\right. \\
& \left.+2 r_{1} r_{2} M_{1} M_{2}\right\} /\left(2 M_{1}+M_{2}\right),
\end{aligned}
$$

由反应讨程中的键级守恒 ${ }^{[1]}, r_{2}$ 可表示为 $r_{1}$ 的函数. $V\left(r_{1}\right)$ 是两个 Lennard-Jones 势. 过渡态的条件是

$\left.\frac{d U}{d r_{1}}\right|_{R_{1}}=0$, 记 $V^{\prime}=\left.\frac{d V}{d r_{1}}\right|_{R_{1}} I^{\prime}-\left.\frac{d I}{d r_{1}}\right|_{R_{1}}$.

$R_{1}$ 是过渡时 $\mathrm{y}$ 原子间的距离, 即临界键长. 平均临界键长为

$$
\bar{R}_{1}-\frac{1}{Q} \int e^{-\frac{V\left(R_{1}\right)}{d T}} F\left(R_{1}\right) R_{1} d R_{1},
$$

式中的 $Q$ 为归一化因子, $F\left(R_{1}\right)$ 由下式确定:

$$
\begin{aligned}
F\left(R_{1}\right)= & \frac{1}{\hbar^{2}}\left[\frac{d^{2} V}{d R_{1}^{2}} \frac{2 I^{2}\left(R_{1}\right)}{I^{\prime}}\right. \\
& +\frac{4 V^{\prime} I\left(R_{1}\right)}{I^{\prime}} \frac{d I\left(R_{1}\right)}{d R_{1}} \\
& \left.-\frac{2 V^{\prime} I^{2}\left(R_{1}\right)}{I^{\prime 2}} \frac{d I^{\prime}}{d R_{1}}\right] .
\end{aligned}
$$

冻络反应坐标时, 过度态的配分函数为

$$
\begin{aligned}
Q^{ \pm}- & {\left[\frac{2 \pi\left(2 M_{1}+M_{2}\right)}{h^{2}} k T\right]^{3 / 2} \frac{8 \pi^{2} I\left(\bar{R}_{1}\right) k T}{h^{2}} } \\
& \prod_{i=1}^{3} \frac{e^{-\frac{A v i}{2 k T}}}{h^{-\frac{h p i}{k T}}}
\end{aligned}
$$

其中的振动频率由文献 [1] 中的方法求得. 反应物的配分函数为

$$
\begin{gathered}
Q=\left[\frac{4 \pi M_{1} k T}{h^{2}}\right]^{3 / 2}\left[\frac{2 \pi M_{2} k T}{h^{2}}\right]^{3 / 2} \\
\cdot \frac{8 \pi^{2} I^{\prime} k T}{2 h^{2}} \frac{e^{-\frac{h \nu^{\prime}}{2 k T}}}{1-e^{-\frac{h \nu^{\prime}}{k T}} Q_{E},}
\end{gathered}
$$

$I^{\prime}$ 是 $y_{2}$ 分子关于其质心的转动惯量, $v^{\prime}$ 是 $y_{2}$ 的振动频率. 电子的配分函数 $Q_{k}-2$. 反应速率常数为

$$
K=\frac{k T}{h} \frac{Q^{ \pm}}{Q} e^{-\frac{V\left(R_{1}\right)-V\left(r_{10}\right)}{k T}},
$$

$V\left(\bar{R}_{1}\right)$ 可认为是过渡态的基态平均能量. $V\left(r_{10}\right)$ 是反应物的基态能量. 由(5)式可得 计算活化能和指前因子的公式如下:

$$
\begin{gathered}
\Delta E=R T^{2} \frac{\partial \ln K}{\partial T}, \\
A=\frac{k T}{h} e^{2} e^{\frac{\Delta s}{R}}, \quad \Delta S-R \frac{\partial}{\partial T}\left[T \ln \frac{Q^{ \pm}}{Q}\right],
\end{gathered}
$$

利用以上理论讨论了下列三种反应: $\mathrm{F}+\mathrm{H}_{2} \longrightarrow \mathrm{FH}+\mathrm{H}, \mathrm{O}+\mathrm{H}_{2} \longrightarrow \mathrm{OH}+\mathrm{H}$ 和 $\mathrm{H}+\mathrm{D}_{2} \longrightarrow \mathrm{HD}+\mathrm{D}$, 计算了它们的平均 临界键长, 以确定过渡态的构型. 也计算了 反应活化能、指前因子和反应速率常数。所 用常数来自文城 $[2,3]$. 计算结果与实验值 符合得很好。

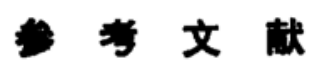

[1] Johnston, H.S. and Goldfinger, P., J. Chem. Phys., 37(1962), 700. 


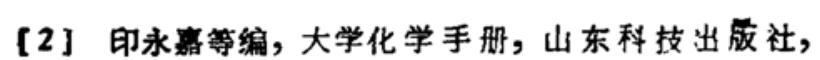
1024; 992-994.

[3] Johnston, H.S. and Parr, C., J. Am. Chem
Soc., 85(1963), 2544.

董顺乐丁世良

(山东大学物理系理论化学研究室, 济南 250100)

\section{高活性钝系加氢催化剂的研究"}

双组分钛系加氢催化剂的研究已有一些 报道, 但绝大部分催化体系的活性较低, 稳定 性很差. 最近我们发现用活性碱金属氢化物 与几种茂钛络合物组成的催化体系, 在常温 常压下对 1-已烯的加氢反应显示出很高的 活性, 例如 $\mathrm{Cp}_{2} \mathrm{~T}_{1} \mathrm{Cl}_{2} / \mathrm{NaH}$ 在 $20^{\circ} \mathrm{C} 、 1 \mathrm{~atm}$ 氢压下对 1-已烯加氢反应的最高活性可达 $2270 \mathrm{molH}_{2} /(\mathrm{molTi} \cdot \mathrm{min})$. 这不仅高于常 温常压下所有的钛系加氢催化剂, 也超过了 常见的 $P d 、 R h 、 R u$ 等贵金属加氢催化剂.

该催化体系的高活性主要应归因于采用 了自制的高活性碱金属氢化物. 市售氢化钠 不能与 $\mathrm{C}_{\mathrm{p}_{2}} \mathrm{TiCl}_{2}$ 组成催化加氢体系; 活性 $\mathrm{NaH}$ 和 $\mathrm{KH}$ 体系具有相近的催化活性和动 力学行为; 活性 $\mathrm{LiH}$ 体系的催化活性要低 得多, 动力学行为也明显有别于前两者. 茂 基对该体系的催化活性有很大的影响, 例 如对于 1-已烯加氢反应有如下活性顺序: $\mathrm{C}_{2} \mathrm{TiCl}_{2}>\mathrm{Cp}_{\mathrm{piCl}} \gg \mathrm{TiCl}_{4}$. 反应动力学 曲线表明, 该催化体系具有非常高的初活性, 但失活很快, $\mathrm{Cp}_{2} \mathrm{TiCl}_{2} / \mathrm{NaH}$ 体系对于 $1-已$ 烯加氢反应在 $30 \mathrm{~min}$ 和 $120 \mathrm{~min}$ 时的催化效 率 (T. O.) 分别为 3250 和 $3830 \mathrm{~mol} \mathrm{H}_{2} /$ mol Ti. 稳定性差是所有钛系双组分均相加 咅催化体系的共同特点, 其原因可能与 $\mathrm{Ti}$ (IV) 物种的还原及二茂钛的二聚反应有关. 我们设想利用大位阻的取代茂基钛氯化物有
可能抑制二茂钛的二聚反应，从而提高体 系的催化效率. 实验结果确实如此, 许多有 很大的空间位阻的取代茂基钛氯化物与活 性碱金属氢化物组成的体系均显示出了很 好的稳定性, 但其初活性有所下降. 例如 ( $\left.><_{\mathrm{Cp}_{\mathrm{p}}}^{\mathrm{C}_{6} \mathrm{H}_{4} \mathrm{OCH}_{3}-\mathrm{P}}\right)_{2} \mathrm{TiCl}_{2} / \mathrm{NaH}$ 对 1-己 烯加氢反应的催化效率（T. O）可达 9290 $\mathrm{mol} \mathrm{H}_{2} / \mathrm{mol} \mathrm{Ti}(80 \mathrm{~min})$, 它的初活性为 692 $\mathrm{mol} \mathrm{H}_{2} /(\mathrm{mol} \mathrm{Ti} \cdot \mathrm{min})$. 这一结果是目前所 见到的钛系加氢催化剂中最高的, 同时也说 明利用配体的空间效应能有效地抑制二茂铲 的二聚反应, 从而得到活性与选择性兼顾的 钛系加氢催化体系. 值得指出的是这类催化 体系在烯烃加氢反应中对温度的依赖性很 小, 例如 $\mathrm{Cp}_{2} \mathrm{TiCl}_{2} / \mathrm{KH}$ 在 $-30^{\circ} \mathrm{C}$ 下对 1己烯加氢反应的初活性仍达到 $1510 \mathrm{~mol} \mathrm{H}_{2} /$ $(\mathrm{mol} \mathrm{Ti} \cdot \mathrm{min})$. 从反应动力学曲线看, 失活 反应在低温下也是一个快反应. 在加氢过程 中, 对于所研究的催化体系, 均未发现明显的 烯烃异构化反应.

张一平 徐等 廖世健

(中国科学院大连化学物理研究所, 大连 116023)

陈寿山

(南开大学元素有机化学研究所, 天浅 30001 )

*国家自然科学基金资助项目.

\section{足球分子及其异构体的电子结构和 UV 谱*}

最近 Kroto 等人 ${ }^{[1]}$ 让激光气化的石墨通 过一个高压超声速喷嘴, 得到了一系列新型
稳定的碳原子簇, 其中 $\mathrm{C}_{60}$ 占绝对优势, 他们 认为它具有笼状结构, 由十二个五元环和二 